\title{
Risks of breast, endometrial, and ovarian cancers after twin births
}

\author{
Jianguang $\mathrm{Ji}^{1}$, Asta Försti ${ }^{1}, 2$, Jan Sundquist ${ }^{1}$ and Kari Hemminki ${ }^{1,2}$ \\ ${ }^{1}$ Karolinska Institute, Center for Family and Community Medicine, Alfred Nobels alle 12, 14183 Huddinge, Sweden \\ ${ }^{2}$ Division of Molecular Genetic Epidemiology, German Cancer Research Center (DKFZ), D-69120 Heidelberg, Germany \\ (Correspondence should be addresses to J Ji; Email: jianguang.ji@ki.se)
}

\begin{abstract}
The concentrations of endogenous hormones differ between women with twin and singleton births, with a possible influence on the risk of cancer. We used the nationwide Swedish FamilyCancer Database, including 30409 women with a twin birth, to examine the subsequent risks of breast, endometrial, and ovarian cancers. Relative risks (RRs) were calculated in a log-linear Poisson regression model of person-years as offset. Cancer data were retrieved from the Swedish Cancer Registry; a total of 1010, 210, and 174 women were diagnosed with breast, endometrial, and ovarian cancers respectively, after a twin birth. A significant decrease in the risk of breast cancer was noted among women with a twin birth compared with women with a singleton birth (RR $0.85,95 \%$ confidence interval $(\mathrm{Cl}) 0.74-0.98)$. The protective effects were observed throughout the intervals after last pregnancy and they were strongest shortly after the last pregnancy in women who delivered a twin birth before 30 years of age. Twin birth did not change the risk of endometrial cancer $(1.08,95 \% \mathrm{Cl} 0.79-1.47)$ but the RR was increased for women with the number of pregnancies $\geq 4(1.39,95 \% \mathrm{Cl} 1.11-1.76)$. The RR for ovarian cancer was $0.95(95 \%$ $\mathrm{Cl} 0.79-1.15)$. Our study showed that twin births significantly reduced the subsequent risk of breast cancer. However, the associations of twin births with endometrial and ovarian cancers were not substantial.
\end{abstract}

Endocrine-Related Cancer (2007) 14 703-711

\section{Introduction}

The physiology of twin and singleton pregnancies differ, as characterized by higher levels of estradiol and testosterone during twin pregnancy, and higher concentrations of follicle-stimulating hormone and sex hormone-binding globulin after twin pregnancy (Thomas et al. 1998, Hall 2003). These changes may influence the occurrence of hormone responsive cancers, such as breast, endometrial, and ovarian cancers (Neale et al. 2004, 2005a,b). A slightly reduced risk of breast cancer among women with multiple births has been reported earlier by Jacobson et al. (1989), but several subsequent studies have failed to confirm this observation (Nasca et al. 1992, Hsieh et al. 1993, Dietz et al. 1995). The possible association of twin births with endometrial and ovarian cancers has been difficult to assess because of the rareness of the affected women (Lambe et al. 1999, Mogren et al. 2001, Neale et al. 2005b). However, a reliable estimation of the consequences of twin pregnancies will help us to better understand hormone-related cancers. To address this issue, a historical cohort study was implemented using the nationwide Swedish Family-Cancer Database, and the risks of breast, endometrial, and ovarian cancers were examined among a total of 30409 women who ever delivered a twin birth.

\section{Materials and methods}

The Family-Cancer Database has been created by linking information from the Multigeneration Register, national censuses, Swedish Cancer Registry and death notifications (Hemminki et al. 2001). Data on family relationships were obtained from the Multigeneration Register, where children born in Sweden in 1932 and later, are registered with their biological parents as families. Twin births were called when two offspring were born at the same time. Women with triplets or high order of multiple births were not included in this 
study; women with multiple twin births were also excluded. Register linkages were carried out using the individually unique national registration number (different from the national identification number). The Swedish Cancer Registry is based on compulsory reports of individual cases provided by physicians (clinical report) and by pathologists or cytologists (pathology report); the coverage of the cancer registration is currently considered to be close to $100 \%$. The database (MigMed2) was updated in 2006 to include the cancer cases from years 1958 to 2004.

Parous women with the last pregnancy recorded in 1958-1989 were included in this study; a total of 30409 women were noted with a twin birth and over 1.5 million women with only singleton births. The ages at pregnancy ranged from 13 to 58 years. The followup was started at last pregnancy, and terminated at the diagnosis of first cancer, death, emigration, or the closing date of the study, December 31, 2004. The mean follow-up time was 28 years, ranging from 0 to 46 years. Only cases classified as primary neoplasms of the breast (International Classification of Diseases 7th revision, code 170), endometrium (172), and ovary (175) were considered. The controls were women with singleton births in the defined parity and time interval class. Relative risks (RRs) and 95\% confidence intervals (CIs) were calculated in a log-linear Poisson regression model of person-years as offset (PROC GENMOD, SAS version 9.2; SAS Institute, Cary, NC, USA), and examined separately for breast, endometrial, and ovarian cancers. The RR was examined separately for mothers of twins with different numbers of pregnancies, using the risk for women with the same number of singleton births as a reference. The risks were adjusted for age (categorical variable in 14 fiveyear categories), period (categorical variable in four categories) and age at first childbirth (four categories, $<20,20-24,25-30$, and $\geq 30$ years). In the analyses of all women with a twin birth, the number of pregnancies was also adjusted. However, the full term and premature births cannot be distinguished in our database: the information about the incomplete pregnancies, such as miscarriages and terminations, was also unavailable. All independent variables were from registered sources containing no missing values.

\section{Results}

Breast cancer was diagnosed in 1010 women with twin births and in 57080 women with only singleton births respectively. Table 1 shows the RRs of breast cancer among women ever delivered a twin birth, using the risk for women with singleton births in the same interval class (e.g. interval after last pregnancy) as reference $(R R=1.00)$. The risks were further analyzed according to the mothers' age at twin birth $(<30$ and $\geq 30$ years) and the order of the twin pregnancy. Mothers with a twin birth had a significant decreased risk of breast cancer, with a RR of 0.85 (95\% CI $0.74-0.98$ ). The RRs ranged from 0.81 (number of pregnancies $=1$ ) to 0.91 (number of pregnancies $\geq 4$ ). The protective effects were observed throughout the intervals after last pregnancy, with few exceptions. Women who delivered a twin birth before 30 years of age had a decreased risk compared with those delivering twin later; the effect was consistent in all pregnancies and interval classes. We also calculated the RRs for all the women whose last pregnancy was a twin pregnancy (data not shown). The RR was 0.87 ( $N=687,95 \%$ CI $0.79-0.95)$; the corresponding RRs for the women who delivered the last twin birth before or after 30 years of age were $0.78(N=267,95 \%$ CI $0.67-0.92)$ and $0.93(N=420,95 \%$ CI $0.82-1.05)$ respectively. The decreased RRs were observed throughout the intervals after the last pregnancy.

Table 2 shows the RRs of endometrial and ovarian cancers among mothers of twins. A total of 210 women were diagnosed with endometrial cancer after a twin birth. The RR was marginally increased compared with women with a singleton birth $(1.08,95 \%$ CI 0.79-1.47). A significant increase was noted for women with the number of pregnancies $\geq 4$, with a RR of 2.18 (95\% CI 1.19-4.01) and 1.61 (95\% CI 1.11-2.19) when the twin pregnancy was the first and middle pregnancy respectively. For ovarian cancer, a total of 174 women were diagnosed after a twin birth with a RR of 0.95 (95\% CI $0.79-1.15)$. There was a decrease in RRs for intervals 0-9 years after last pregnancy but the RR was significant only for pregnancy of one and when these intervals were combined $(N=10, \mathrm{RR}=0.47,95 \%$ CI $0.26-0.84)$. One RR (2.33) was increased for women with the number of pregnancies $\geq 4$.

\section{Discussion}

The prevalence of twin births has increased greatly since 1980s because of frequent usage of the assisted reproduction techniques and fertility drugs (Herskind et al. 2005, Toledo 2005). The concentrations of endogenous hormones among women with twin births are known to differ from mothers of singletons. Thus, reliable cancer risk estimation in women following twin births can help to uncover hormonal effects on cancer development. A total of 30409 mothers who ever delivered a twin birth were included in the present 
Table 1 Relative risk of breast cancer among women with a twin birth

\begin{tabular}{|c|c|c|c|c|c|c|c|c|c|c|c|c|c|}
\hline \multirow[b]{3}{*}{ Parameter } & \multicolumn{12}{|c|}{ Age at twin birth } & \multirow[b]{3}{*}{ Singleton reference } \\
\hline & \multicolumn{4}{|c|}{ All } & \multicolumn{4}{|c|}{$<30$} & \multicolumn{4}{|c|}{$\geq 30$} & \\
\hline & $N$ & RR & \multicolumn{2}{|c|}{$95 \% \mathrm{Cl}$} & $N$ & RR & \multicolumn{2}{|c|}{$95 \% \mathrm{Cl}$} & $N$ & RR & \multicolumn{2}{|c|}{$95 \% \mathrm{Cl}$} & \\
\hline \multicolumn{14}{|c|}{ Intervals after last pregnancy (years) } \\
\hline Total & 1010 & 0.85 & 0.74 & 0.98 & 501 & 0.79 & 0.65 & 0.97 & 509 & 0.91 & 0.74 & 1.12 & 57080 \\
\hline \multicolumn{14}{|c|}{ Number of pregnancies $=1$} \\
\hline $0-9$ & 15 & 0.74 & 0.44 & 1.24 & 5 & 0.65 & 0.25 & 1.72 & 10 & 0.79 & 0.43 & 1.45 & 1068 \\
\hline $10-19$ & 42 & 0.75 & 0.54 & 1.04 & 17 & 0.65 & 0.37 & 1.14 & 25 & 0.84 & 0.51 & 1.38 & 2884 \\
\hline $20-$ & 111 & 0.84 & 0.65 & 1.08 & 72 & 0.82 & 0.59 & 1.13 & 39 & 0.89 & 0.56 & 1.41 & 7497 \\
\hline All & 168 & 0.81 & 0.67 & 0.98 & 94 & 0.76 & 0.57 & 1.00 & 74 & 0.88 & 0.66 & 1.19 & 11449 \\
\hline \multicolumn{14}{|c|}{ Number of pregnancies $=2$, total } \\
\hline $0-9$ & 41 & 0.87 & 0.62 & 1.22 & 20 & 0.99 & 0.63 & 1.56 & 21 & 0.78 & 0.48 & 1.28 & 2564 \\
\hline $10-19$ & 130 & 0.98 & 0.76 & 1.28 & 61 & 0.90 & 0.61 & 1.32 & 69 & 1.07 & 0.73 & 1.56 & 7488 \\
\hline $20-$ & 243 & 0.87 & 0.78 & 0.96 & 143 & 0.80 & 0.69 & 0.93 & 100 & 0.98 & 0.81 & 1.18 & 16843 \\
\hline All & 414 & 0.90 & 0.76 & 1.06 & 224 & 0.83 & 0.66 & 1.05 & 190 & 1.00 & 0.78 & 1.28 & 26895 \\
\hline \multicolumn{14}{|c|}{ Twin first pregnancy } \\
\hline $0-9$ & 12 & 0.81 & 0.45 & 1.46 & 9 & 0.81 & 0.41 & 1.60 & 3 & 0.81 & 0.26 & 2.52 & 2564 \\
\hline $10-19$ & 38 & 0.97 & 0.61 & 1.55 & 28 & 0.88 & 0.51 & 1.51 & 10 & 1.39 & 0.52 & 3.70 & 7488 \\
\hline $20-$ & 52 & 0.67 & 0.51 & 0.88 & 47 & 0.70 & 0.53 & 0.91 & 5 & 0.49 & 0.21 & 1.17 & 16843 \\
\hline All & 102 & 0.78 & 0.56 & 1.10 & 84 & 0.76 & 0.53 & 1.11 & 18 & 0.87 & 0.39 & 1.94 & 26895 \\
\hline \multicolumn{14}{|c|}{ Twin last pregnancy } \\
\hline $0-9$ & 29 & 0.90 & 0.61 & 1.32 & 11 & 1.21 & 0.63 & 2.32 & 18 & 0.78 & 0.47 & 1.30 & 2564 \\
\hline $10-19$ & 92 & 0.99 & 0.73 & 1.34 & 33 & 0.92 & 0.53 & 1.59 & 59 & 1.03 & 0.68 & 1.57 & 7488 \\
\hline $20-$ & 191 & 0.94 & 0.84 & 1.05 & 96 & 0.86 & 0.73 & 1.02 & 95 & 1.03 & 0.85 & 1.24 & 16843 \\
\hline All & 312 & 0.95 & 0.79 & 1.14 & 140 & 0.88 & 0.66 & 1.17 & 172 & 1.01 & 0.78 & 1.32 & 26895 \\
\hline \multicolumn{14}{|c|}{ Number of pregnancies $=3$, total } \\
\hline $0-9$ & 36 & 1.03 & 0.76 & 1.39 & 10 & 0.73 & 0.41 & 1.30 & 26 & 1.22 & 0.87 & 1.71 & 1556 \\
\hline $10-19$ & 69 & 0.81 & 0.62 & 1.05 & 30 & 0.79 & 0.52 & 1.20 & 39 & 0.82 & 0.58 & 1.16 & 3978 \\
\hline $20-$ & 145 & 0.85 & 0.71 & 1.02 & 74 & 0.82 & 0.63 & 1.05 & 71 & 0.89 & 0.64 & 1.24 & 7590 \\
\hline All & 250 & 0.86 & 0.73 & 1.01 & 114 & 0.79 & 0.63 & 1.00 & 136 & 0.92 & 0.75 & 1.15 & 13124 \\
\hline \multicolumn{14}{|c|}{ Twin first pregnancy } \\
\hline $0-9$ & 5 & 0.91 & 0.45 & 1.85 & 3 & 0.63 & 0.26 & 1.54 & 2 & 2.77 & 0.88 & 8.74 & 1556 \\
\hline $10-19$ & 8 & 0.63 & 0.32 & 1.24 & 7 & 0.61 & 0.30 & 1.24 & 1 & 0.86 & 0.11 & 6.41 & 3978 \\
\hline $20-$ & 24 & 0.95 & 0.59 & 1.53 & 20 & 0.84 & 0.50 & 1.43 & 4 & 2.59 & 0.66 & 10.18 & 7590 \\
\hline All & 37 & 0.85 & 0.57 & 1.27 & 30 & 0.75 & 0.49 & 1.16 & 7 & 2.06 & 0.83 & 5.13 & 13124 \\
\hline \multicolumn{14}{|c|}{ Twin middle pregnancy } \\
\hline $0-9$ & 12 & 1.26 & 0.74 & 2.15 & 5 & 0.80 & 0.36 & 1.78 & 7 & 2.18 & 1.09 & 4.36 & 1556 \\
\hline $10-19$ & 17 & 0.76 & 0.43 & 1.34 & 14 & 0.86 & 0.48 & 1.54 & 3 & 0.48 & 0.14 & 1.67 & 3978 \\
\hline $20-$ & 52 & 1.14 & 0.82 & 1.58 & 35 & 1.02 & 0.69 & 1.51 & 17 & 1.52 & 0.84 & 2.74 & 7590 \\
\hline All & 81 & 1.05 & 0.78 & 1.41 & 54 & 0.95 & 0.67 & 1.35 & 27 & 1.33 & 0.81 & 2.17 & 13124 \\
\hline
\end{tabular}




\begin{tabular}{|c|c|c|c|c|c|c|c|c|c|c|c|c|c|}
\hline \multirow[b]{3}{*}{ Parameter } & \multicolumn{12}{|c|}{ Age at twin birth } & \multirow[b]{3}{*}{ Singleton reference } \\
\hline & \multicolumn{4}{|c|}{ All } & \multicolumn{4}{|c|}{$<30$} & \multicolumn{4}{|c|}{$\geq 30$} & \\
\hline & $N$ & $\mathrm{RR}$ & \multicolumn{2}{|c|}{$95 \% \mathrm{Cl}$} & $N$ & $\mathrm{RR}$ & \multicolumn{2}{|c|}{$95 \% \mathrm{Cl}$} & $N$ & $\mathrm{RR}$ & \multicolumn{2}{|c|}{$95 \% \mathrm{Cl}$} & \\
\hline Twin las & & & & & & & & & & & & & \\
\hline $0-9$ & 19 & 0.95 & 0.65 & 1.40 & 2 & 0.76 & 0.22 & 2.66 & 17 & 0.98 & 0.65 & 1.49 & 1556 \\
\hline $10-19$ & 44 & 0.87 & 0.66 & 1.16 & 9 & 0.87 & 0.44 & 1.72 & 35 & 0.87 & 0.61 & 1.24 & 3978 \\
\hline $20-$ & 69 & 0.69 & 0.54 & 0.89 & 19 & 0.58 & 0.34 & 1.00 & 50 & 0.75 & 0.51 & 1.09 & 7590 \\
\hline All & 132 & 0.78 & 0.63 & 0.96 & 30 & 0.64 & 0.41 & 1.00 & 102 & 0.83 & 0.65 & 1.06 & 13124 \\
\hline \multicolumn{14}{|c|}{ Number of pregnancies $\geq 4$, total } \\
\hline $0-9$ & 18 & 0.65 & 0.39 & 1.07 & 7 & 0.67 & 0.33 & 1.40 & 11 & 0.63 & 0.33 & 1.19 & 792 \\
\hline $10-19$ & 53 & 1.02 & 0.77 & 1.36 & 19 & 0.90 & 0.57 & 1.43 & 34 & 1.11 & 0.77 & 1.60 & 1531 \\
\hline $20-$ & 107 & 0.93 & 0.79 & 1.08 & 43 & 0.82 & 0.67 & 1.00 & 64 & 1.02 & 0.86 & 1.21 & 3289 \\
\hline All & 178 & 0.91 & 0.77 & 1.09 & 69 & 0.82 & 0.62 & 1.07 & 109 & 0.99 & 0.80 & 1.22 & 5612 \\
\hline \multicolumn{14}{|c|}{ Twin first pregnancy } \\
\hline $0-9$ & 2 & 0.72 & 0.23 & 2.26 & 2 & 0.81 & 0.25 & 2.61 & 0 & & & & 792 \\
\hline $10-19$ & 8 & 1.62 & 0.84 & 3.11 & 7 & 1.55 & 0.75 & 3.17 & 1 & 2.46 & 0.33 & 18.19 & 1531 \\
\hline $20-$ & 10 & 0.99 & 0.70 & 1.40 & 10 & 1.04 & 0.73 & 1.47 & 0 & & & & 3289 \\
\hline All & 20 & 1.13 & 0.71 & 1.78 & 19 & 1.14 & 0.71 & 1.84 & 1 & 0.89 & 0.12 & 6.71 & 5612 \\
\hline \multicolumn{14}{|c|}{ Twin middle pregnancy } \\
\hline $0-9$ & 7 & 0.49 & 0.25 & 0.96 & 5 & 0.68 & 0.31 & 1.48 & 2 & 0.29 & 0.08 & 1.05 & 792 \\
\hline $10-19$ & 22 & 0.86 & 0.56 & 1.33 & 11 & 0.74 & 0.41 & 1.35 & 11 & 1.02 & 0.53 & 1.96 & 1531 \\
\hline $20-$ & 54 & 0.93 & 0.79 & 1.10 & 31 & 0.86 & 0.68 & 1.08 & 23 & 1.04 & 0.85 & 1.27 & 3289 \\
\hline All & 83 & 0.85 & 0.67 & 1.08 & 47 & 0.81 & 0.59 & 1.10 & 36 & 0.91 & 0.63 & 1.32 & 5612 \\
\hline \multicolumn{14}{|c|}{ Twin last pregnancy } \\
\hline $0-9$ & 9 & 0.83 & 0.44 & 1.57 & 0 & & & & 9 & 0.87 & 0.45 & 1.71 & 792 \\
\hline $10-19$ & 23 & 1.08 & 0.72 & 1.61 & 1 & 0.53 & 0.08 & 3.60 & 22 & 1.13 & 0.73 & 1.77 & 1531 \\
\hline $20-$ & 43 & 0.91 & 0.72 & 1.14 & 2 & 0.29 & 0.15 & 0.55 & 41 & 1.02 & 0.81 & 1.28 & 3289 \\
\hline All & 75 & 0.94 & 0.73 & 1.22 & 3 & 0.31 & 0.10 & 0.94 & 72 & 1.03 & 0.78 & 1.35 & 5612 \\
\hline
\end{tabular}

$\mathrm{N}$, observed cases; RR, relative risk; Bold type, $95 \% \mathrm{Cl}$ does not include 1.00 . 
Table 2 Relative risk of endometrial and ovarian cancers among women with a twin birth

\begin{tabular}{|c|c|c|c|c|c|c|c|c|c|c|}
\hline \multirow[b]{2}{*}{ Parameter } & \multicolumn{5}{|c|}{ Endometrial cancer } & \multicolumn{5}{|c|}{ Ovarian cancer } \\
\hline & $N$ & $\mathrm{RR}$ & \multicolumn{2}{|c|}{$95 \% \mathrm{Cl}$} & Singleton reference & $N$ & $\mathrm{RR}$ & \multicolumn{2}{|c|}{$95 \% \mathrm{Cl}$} & Singleton reference \\
\hline \multicolumn{11}{|c|}{ Intervals after last pregnancy (years) } \\
\hline Total & 210 & 1.08 & 0.79 & 1.47 & 9001 & 174 & 0.95 & 0.79 & 1.15 & 8677 \\
\hline \multicolumn{11}{|c|}{ Number of pregnancies $=1$} \\
\hline $0-9$ & 1 & 1.26 & 0.23 & 6.89 & 49 & 1 & 0.22 & 0.06 & 0.77 & 248 \\
\hline $10-19$ & 5 & 1.12 & 0.48 & 2.61 & 252 & 6 & 0.69 & 0.35 & 1.34 & 467 \\
\hline $20-$ & 23 & 0.89 & 0.71 & 1.13 & 1476 & 24 & 1.20 & 0.94 & 1.54 & 1142 \\
\hline All & 29 & 0.93 & 0.59 & 1.48 & 1777 & 31 & 0.93 & 0.67 & 1.29 & 1857 \\
\hline \multicolumn{11}{|c|}{ Number of pregnancies $=2$, total } \\
\hline $0-9$ & 2 & 1.58 & 0.70 & 3.60 & 67 & 5 & 0.71 & 0.35 & 1.42 & 404 \\
\hline $10-19$ & 9 & 0.89 & 0.48 & 1.65 & 548 & 13 & 0.72 & 0.43 & 1.21 & 1025 \\
\hline $20-$ & 64 & 1.10 & 0.77 & 1.59 & 3396 & 49 & 1.18 & 0.90 & 1.55 & 2476 \\
\hline All & 75 & 1.08 & 0.77 & 1.50 & 4011 & 67 & 1.00 & 0.77 & 1.29 & 3905 \\
\hline \multicolumn{11}{|c|}{ Twin first pregnancy } \\
\hline $0-9$ & 2 & 4.57 & 1.87 & 11.19 & 67 & 1 & 0.48 & 0.14 & 1.69 & 404 \\
\hline $10-19$ & 5 & 1.55 & 0.60 & 4.01 & 548 & 5 & 0.95 & 0.48 & 1.87 & 1025 \\
\hline $20-$ & 12 & 0.74 & 0.31 & 1.75 & 3396 & 13 & 1.13 & 0.70 & 1.83 & 2476 \\
\hline All & 19 & 0.93 & 0.52 & 1.66 & 4011 & 19 & 0.99 & 0.65 & 1.51 & 3905 \\
\hline \multicolumn{11}{|c|}{ Twin last pregnancy } \\
\hline $0-9$ & 0 & & & & 67 & 4 & 0.80 & 0.37 & 1.76 & 404 \\
\hline $10-19$ & 4 & 0.58 & 0.21 & 1.63 & 548 & 8 & 0.63 & 0.32 & 1.23 & 1025 \\
\hline $20-$ & 52 & 1.25 & 0.83 & 1.87 & 3396 & 36 & 1.20 & 0.88 & 1.62 & 2476 \\
\hline All & 56 & 1.13 & 0.81 & 1.59 & 4011 & 48 & 1.00 & 0.77 & 1.31 & 3905 \\
\hline \multicolumn{11}{|c|}{ Number of pregnancies $=3$, total } \\
\hline $0-9$ & 1 & 0.97 & 0.16 & 5.84 & 43 & 3 & 0.55 & 0.24 & 1.26 & 246 \\
\hline $10-19$ & 8 & 0.92 & 0.50 & 1.68 & 378 & 13 & 1.09 & 0.69 & 1.74 & 541 \\
\hline $20-$ & 41 & 1.08 & 0.78 & 1.49 & 1661 & 29 & 1.10 & 0.84 & 1.46 & 1157 \\
\hline All & 50 & 1.04 & 0.72 & 1.52 & 2082 & 45 & 1.03 & 0.75 & 1.41 & 1944 \\
\hline \multicolumn{11}{|c|}{ Twin first pregnancy } \\
\hline $0-9$ & 1 & 5.54 & 0.91 & 33.77 & 43 & 1 & 1.17 & 0.37 & 3.73 & 356 \\
\hline $10-19$ & 0 & & & & 378 & 1 & 0.56 & 0.12 & 2.59 & 816 \\
\hline $20-$ & 6 & 1.06 & 0.45 & 2.47 & 1661 & 4 & 1.03 & 0.48 & 2.19 & 1743 \\
\hline All & 7 & 0.95 & 0.35 & 2.55 & 2082 & 6 & 0.91 & 0.40 & 2.07 & 2915 \\
\hline \multicolumn{11}{|c|}{ Twin middle pregnancy } \\
\hline $0-9$ & 0 & & & & 43 & 0 & & & & 356 \\
\hline $10-19$ & 3 & 1.21 & 0.47 & 3.16 & 378 & 3 & 0.94 & 0.35 & 2.50 & 816 \\
\hline $20-$ & 12 & 1.17 & 0.68 & 2.03 & 1661 & 10 & 1.42 & 0.89 & 2.27 & 1743 \\
\hline All & 15 & 1.14 & 0.61 & 2.14 & 2082 & 13 & 1.10 & 0.61 & 1.98 & 2915 \\
\hline
\end{tabular}




\begin{tabular}{|c|c|c|c|c|c|c|c|c|c|c|}
\hline \multirow[b]{2}{*}{ Parameter } & \multicolumn{5}{|c|}{ Endometrial cancer } & \multicolumn{5}{|c|}{ Ovarian cancer } \\
\hline & $N$ & $\mathrm{RR}$ & \multicolumn{2}{|c|}{$95 \% \mathrm{Cl}$} & Singleton reference & $N$ & $\mathrm{RR}$ & \multicolumn{2}{|c|}{$95 \% \mathrm{Cl}$} & Singleton reference \\
\hline \multicolumn{11}{|c|}{ Twin last pregnancy } \\
\hline $0-9$ & 0 & & & & 43 & 2 & 0.64 & 0.23 & 1.75 & 356 \\
\hline $10-19$ & 5 & 1.03 & 0.46 & 2.29 & 378 & 9 & 1.30 & 0.77 & 2.19 & 816 \\
\hline $20-$ & 23 & 1.04 & 0.69 & 1.57 & 1661 & 15 & 0.98 & 0.66 & 1.46 & 1743 \\
\hline All & 28 & 1.02 & 0.64 & 1.65 & 2082 & 26 & 1.02 & 0.69 & 1.53 & 2915 \\
\hline \multicolumn{11}{|c|}{ Number of pregnancies $\geq 4$, total } \\
\hline $0-9$ & 1 & 1.00 & 0.44 & 2.29 & 25 & 1 & 0.26 & 0.06 & 1.16 & 356 \\
\hline $10-19$ & 11 & 1.39 & 1.06 & 1.82 & 221 & 15 & 1.55 & 1.06 & 2.26 & 816 \\
\hline $20-$ & 44 & 1.41 & 1.04 & 1.92 & 885 & 15 & 0.72 & 0.52 & 1.00 & 1743 \\
\hline All & 56 & 1.39 & 1.11 & 1.76 & 1131 & 31 & 0.90 & 0.67 & 1.22 & 2915 \\
\hline \multicolumn{11}{|c|}{ Twin first pregnancy } \\
\hline $0-9$ & 0 & & & & 25 & 0 & & & & 356 \\
\hline $10-19$ & 1 & 1.32 & 0.56 & 3.06 & 221 & 1 & 1.10 & 0.30 & 3.97 & 816 \\
\hline $20-$ & 7 & 2.57 & 1.22 & 5.43 & 885 & 2 & 1.10 & 0.50 & 2.43 & 1743 \\
\hline All & 8 & 2.18 & 1.19 & 4.01 & 1131 & 3 & 0.95 & 0.41 & 2.23 & 2915 \\
\hline \multicolumn{11}{|c|}{ Twin middle pregnancy } \\
\hline $0-9$ & 1 & 1.74 & 0.74 & 4.08 & 25 & 1 & 0.51 & 0.11 & 2.28 & 356 \\
\hline $10-19$ & 9 & 2.22 & 1.61 & 3.05 & 221 & 5 & 1.02 & 0.54 & 1.92 & 816 \\
\hline $20-$ & 23 & 1.46 & 0.97 & 2.20 & 885 & 6 & 0.57 & 0.38 & 0.87 & 1743 \\
\hline All & 33 & 1.61 & 1.19 & 2.19 & 1131 & 12 & 0.69 & 0.43 & 1.10 & 2915 \\
\hline \multicolumn{11}{|c|}{ Twin last pregnancy } \\
\hline $0-9$ & 0 & & & & 25 & 0 & & & & 356 \\
\hline $10-19$ & 1 & 0.32 & 0.14 & 0.75 & 221 & 9 & 2.33 & 1.42 & 3.82 & 816 \\
\hline $20-$ & 37 & 1.10 & 0.63 & 1.91 & 885 & 7 & 0.83 & 0.54 & 1.26 & 1743 \\
\hline All & 38 & 0.94 & 0.58 & 1.51 & 1131 & 16 & 1.16 & 0.77 & 1.76 & 2915 \\
\hline
\end{tabular}

$\mathrm{N}$, observed cases; RR, relative risk; Bold type, $95 \% \mathrm{Cl}$ does not include 1.00 . 
study, which, to our knowledge, was the largest one on this issue, guaranteeing a reasonable statistical power. Another advantage is that all the cancer cases were retrieved from the Swedish Cancer Registry with a complete national coverage. The present study included the whole population covered by the nationwide registers, and it was thus not influenced by selection or recall bias. However, as a limitation, hormonally relevant factors, such as abortions, use of contraceptive pills or hormone replacement therapy, were unavailable in our database, which may confound our results. However, the associations between these factors and the delivery of twins have not been observed (Bortolus et al. 1999).

When interpreting the results, it is important to note that all comparisons were done between twin and singleton pregnancies. In singleton pregnancies, parity is known to be a protective factor for the three cancers studied (Tavassoli \& Devilee 2003); young age at first childbirth is protective for breast cancer but may be a risk factor for endometrial and ovarian cancers.

The present study indicates that mothers of twins are at a decreased risk of breast cancer, in agreement with some (Wyshak et al. 1983, Jacobson et al. 1989, Albrektsen et al. 1995, Neale et al. 2005b) but not all (Dietz et al. 1995, Mogren et al. 2001, Neale et al. 2004) earlier reports; even an increased risk has been previously reported (Hsieh et al. 1993). The protective effects were consistent in all parity classes, further corroborating the findings. The decreased risk appeared to be more pronounced when women delivered the twins at an age below 30 years and it was particularly strong for these women with the number of pregnancies three or more, when the twin pregnancy was the last one for the women. Our study also showed that the order of twin pregnancies was not an important modifying factor for the decreased risk of breast cancer. Some hypotheses have been put forward to explain the protective effect of twin pregnancy on breast cancer, such as higher concentration of sex hormone-binding globulin (Murphy et al. 1990) and higher level of $\alpha$-fetoproteins with anti-estrogenic effects in women bearing twins (Jacobson et al. 1989); the latter has been supported by an animal model (Jacobson et al. 1989). Similar to the twin pregnancy, pre-eclampsia has been associated with a decreased breast cancer risk. The decreased risk may be explained by increased progesterone levels compared with a normal pregnancy (Peck et al. 2002, Tamimi et al. 2003). In contrast to pre-eclampsia, where estrogen levels are decreased, a twin pregnancy increases the level. The simultaneous decrease of the level of sex hormone-binding globulin in pre-eclampsia and the increase of its level during a twin pregnancy may partly compensate the effects of estrogens (Tamimi et al. 2003). It is noteworthy that the observed risk was decreased throughout the intervals after last pregnancy, in disagreement with the hypothesis that a higher level of estrogen during twin pregnancies will increase the breast cancer risk transiently.

It has been hypothesized that a high level of progesterone during a twin pregnancy will reduce the subsequent risk of endometrial cancer as progesterone inhibits endometrial cell division (Key 1995). A decrease in the risk of endometrial cancer has been observed in an earlier study on the Utah Population Database (Neale et al. 2004). However, the protective effect was present only for the women who had more than three children and the number of affected women was limited (19 cases), weakening the conclusion. Instead, our data showed that the risk of endometrial cancer did not differ between women with twins and singleton births, which was in agreement with an earlier Swedish study (Mogren et al. 2001). A significant increase was even noted for women with the number of pregnancies $\geq 4$ when the twin pregnancy was not the last pregnancy. Obesity has been strongly associated with an increased risk of endometrial cancer (Friedenreich et al. 2007). The concentration of insulin-like growth factor-II (IGF-II) has been reported to be higher among obese individuals than among lean ones (Frystyk et al. 1999). Increased level of IGF-II has also been associated with endometrial cancer (Petridou et al. 2003, Oh et al. 2004), which could partly explain our observation.

A small decrease in the risk of ovarian cancer among women with a twin pregnancy has been reported in some earlier studies (Lambe et al. 1999, Whiteman et al. 2000, Neale et al. 2005b), and the elevated level of progesterone has been suggested to be related to the decrease by inducing apoptosis of transformed ovarian cells. In the present study, a marginally decreased risk was noted, but the overall effect was not significant. The risk of ovarian cancer was significantly decreased in the first interval (0-9 years) after the last pregnancy, which was in agreement with an earlier observation that the protective effect induced by pregnancy would reach its maximum at 2-3 years after the birth and then diminish (Liu et al. 2006). For women with the number of pregnancies $\geq 4$, a significant increase in the risk of ovarian cancer was observed, but it was present only when the twin pregnancy was the last one and only for interval of 10-19 years since the last pregnancy, thus a chance finding could be possible. 
In conclusion, our study showed that women who ever delivered a twin birth had a significant decrease in the risk of breast cancer, and the decrease was consistent in each parity class and all intervals after last pregnancy. No consistent associations were noted for endometrial and ovarian cancers; women with high parity had an increased risk of endometrial cancer; the risk of ovarian cancer was transiently decreased after a twin pregnancy.

\section{Acknowledgements}

This work was supported by Deutsche Krebshilfe, the Swedish Cancer Society, the EU, LSHC-CT-2004503465, and the Swedish Council for Working Life and Social Research. The Family-Cancer Database was created by linking registers maintained at Statistics Sweden and the Swedish Cancer Registry. The authors declare that there is no conflict of interest that would prejudice the impartiality of this scientific work.

\section{References}

Albrektsen G, Heuch I \& Kvale G 1995 Multiple births, sex of children and subsequent breast-cancer risk for the mothers: a prospective study in Norway. International Journal of Cancer 60 341-344.

Bortolus R, Parazzini F, Chatenoud L, Benzi G, Bianchi MM \& Marini A 1999 The epidemiology of multiple births. Human Reproduction Update 5 179-187.

Dietz AT, Newcomb PA, Storer BE, Longnecker MP \& Mittendorf R 1995 Multiple births and risk of breast cancer. International Journal of Cancer 62 162-164.

Friedenreich C, Cust A, Lahmann PH, Steindorf K, BoutronRuault MC, Clavel-Chapelon F, Mesrine S, Linseisen J, Rohrmann S, Boeing H et al. 2007 Anthropometric factors and risk of endometrial cancer: the European prospective investigation into cancer and nutrition. Cancer Causes and Control 18 399-413.

Frystyk J, Skjaerbaek C, Vestbo E, Fisker S \& Orskov H 1999 Circulating levels of free insulin-like growth factors in obese subjects: the impact of type 2 diabetes. Diabetes Metabolism Research and Reviews 15 314-322.

Hall JG 2003 Twinning. Lancet 362 735-743.

Hemminki K, Li X, Plna K, Granström C \& Vaittinen P 2001 The nation-wide Swedish family-cancer database: updated structure and familial rates. Acta Oncologica 40 772-777.

Herskind AM, Basso O, Olsen J, Skytthe A \& Christensen K 2005 Is the natural twinning rate still declining? Epidemiology 16 591-592.

Hsieh CC, Goldman M, Pavia M, Ekbom A, Petridou E, Adami HO \& Trichopoulos D 1993 Breast cancer risk in mothers of multiple births. International Journal of Cancer 54 81-84.
Jacobson HI, Thompson WD \& Janerich DT 1989 Multiple births and maternal risk of breast cancer. American Journal of Epidemiology 129 865-873.

Key TJ 1995 Hormones and cancer in humans. Mutation Research 333 59-67.

Lambe M, Wuu J, Rossing MA \& Hsieh CC 1999 Twinning and maternal risk of ovarian cancer. Lancet 3531941.

Liu Q, Lambe M, Baik I, Cnattingius S, Riman T, Ekbom A, Adami HO \& Hsieh CC 2006 A prospective study of the transient decrease in ovarian cancer risk following childbirth. Cancer Epidemiology Biomarkers and Prevention 15 2508-2513.

Mogren I, Stenlund H \& Hogberg U 2001 Long-term impact of reproductive factors on the risk of cervical, endometrial, ovarian and breast cancer. Acta Oncologica 40 849-854.

Murphy M, Key T, Wang D, Moore J, Clark G \& Allen D 1990 Re: 'Multiple births and maternal risk of breast cancer'. American Journal of Epidemiology 132 199-201.

Nasca PC, Weinstein A, Baptiste M \& Mahoney M 1992 The relation between multiple births and maternal risk of breast cancer. American Journal of Epidemiology 136 1316-1320.

Neale RE, Purdie DM, Murphy MF, Mineau GP, Bishop T \& Whiteman DC 2004 Twinning and the incidence of breast and gynecological cancers (United States). Cancer Causes and Control 15 829-835.

Neale RE, Mineau G, Whiteman DC, Brownbill PA \& Murphy MF 2005a Childhood and adult cancer in twins: evidence from the Utah genealogy. Cancer Epidemiology Biomarkers and Prevention 14 1236-1240.

Neale RE, Darlington S, Murphy MF, Silcocks PB, Purdie DM \& Talback M $2005 b$ The effects of twins, parity and age at first birth on cancer risk in Swedish women. Twin Research and Human Genetics 8 156-162.

Oh JC, Wu W, Tortolero-Luna G, Broaddus R, Gershenson DM, Burke TW, Schmandt R \& Lu KH 2004 Increased plasma levels of insulin-like growth factor 2 and insulinlike growth factor binding protein 3 are associated with endometrial cancer risk. Cancer Epidemiology Biomarkers and Prevention 13 748-752.

Peck JD, Hulka BS, Poole C, Savitz DA, Baird D \& Richardson BE 2002 Steroid hormone levels during pregnancy and incidence of maternal breast cancer. Cancer Epidemiology Biomarkers and Prevention 11 361-368.

Petridou E, Koukoulomatis P, Alexe DM, Voulgaris Z, Spanos E \& Trichopoulos D 2003 Endometrial cancer and the IGF system: a case-control study in Greece. Oncology 64 341-345.

Tamimi R, Lagiou P, Vatten LJ, Mucci L, Trichopoulos D, Hellerstein S, Ekbom A, Adami HO \& Hsieh CC 2003 Pregnancy hormones, pre-eclampsia, and implications for breast cancer risk in the offspring. Cancer Epidemiology Biomarkers and Prevention 12 647-650.

Tavassoli F \& Devilee P 2003 Tumours of the Breast and Female Genital Organs. Pathology and Genetics. Lyon: IARC. 
Thomas HV, Murphy MF, Key TJ, Fentiman IS, Allen DS \& Kinlen LJ 1998 Pregnancy and menstrual hormone levels in mothers of twins compared to mothers of singletons. Annals of Human Biology 25 69-75.

Toledo MG 2005 Is there increased monozygotic twinning after assisted reproductive technology? Australian \& New Zealand Journal of Obstetrics and Gynaecology 45 360-364.
Whiteman DC, Murphy MF, Cook LS, Cramer DW, Hartge P, Marchbanks PA, Nasca PC, Ness RB, Purdie DM \& Risch HA 2000 Multiple births and risk of epithelial ovarian cancer. Journal of National Cancer Institute 92 1172-1177. Wyshak G, Honeyman MS, Flannery JT \& Beck AS 1983 Cancer in mothers of dizygotic twins. Journal of National Cancer Institute 70 593-599. 